\title{
Quasi-Spin-Charge Separation and the Spin Quantum Hall Effect
}

\author{
D.Bernard ${ }^{1} *$ and A. LeClair ${ }^{1,2}$ \\ ${ }^{1}$ Service de Physique Theorique de Saclay, F-91191, Gif-sur-Yvette, France \\ ${ }^{2}$ Newman Laboratory, Cornell University, Ithaca, NY 14853.
}

(February 29, 2000)

\begin{abstract}
We use quantum field theory methods to study the network model for the spin quantum Hall transition. When the couplings are fine tuned in a certain way, the spin and charge degrees of freedom, corresponding to the supercurrent algebras $s u(2)_{0}$ and $\operatorname{ssp}(2 \mid 2)_{-2}$ respectively, decouple in the renormalization group flow. The infrared fixed point of this simpler theory is the coset $\operatorname{osp}(4 \mid 4)_{1} / s u(2)_{0}$ which is closely related to the current algebra $\operatorname{osp}(2 \mid 2)_{-2}$ but not identical. Some critical exponents are computed and shown to agree with the recent predictions based on percolation.
\end{abstract}

${ }^{*}$ Member of the C.N.R.S. 


\section{INTRODUCTION}

Recently a number of works have appeared investigating a new universality class of delocalization transition referred to as the spin quantum Hall effect. This transition can occur in certain dirty superconductors with unbroken $s u(2)$ spin-rotation symmetry. Kagalovsky et. al. constructed an $s u(2)$ invariant network model for the transition and numerically determined some critical exponents [1]. Senthil et. al. on the other hand modeled the phenomena with a supersymmetric spin chain [2,3]. Remarkably, the network model was mapped directly on the lattice onto classical percolation by Gruzberg et. al. and exact exponents were computed which agreed very well with the numerical simulations of the super spin chain 《4.

In the quantum field theory approach to delocalization transitions, the computation of critical exponents is normally a difficult strong-coupling problem requiring the existence of non-trivial infrared fixed points. The claim that for the spin quantum Hall effect the infrared fixed point of the disorder averaged effective field theory is simply percolation is rather unexpected and for this reason we set out to understand this using quantum field theory methods. Our starting point is the hamiltonian formulation of the network model given in [1]. We carry out the disorder averaging using the supersymmetric method in conjunction with conformal field theory methods. This leads to an effective action which consists of a conformal field theory with an osp(4|4) super-current algebra symmetry perturbed by certain marginal operators which are bilinear in the supercurrents.

The one-loop renormalization group ( $R G$ ) $\beta$ eta functions we compute for the three independent couplings appear to be as complicated as those for the usual quantum Hall transition [6]. There exists a fine-tuning of the network model couplings wherein two of the couplings are essentially identified and the resulting model is remarkably simpler and can be solved. This is due in part to the fact that the stress tensor for the $\operatorname{ssp}(4 \mid 4)$ current algebra can be written as the sum of the stress tensors for the $\operatorname{sep}(2 \mid 2)$ level -2 and $s u(2)$ level 0 current algebras corresponding to the charge and spin degrees of freedom respectively. This "spincharge separation" is present in the disorder-averaged effective theory and this leads to two decoupled $\beta$ eta functions. This simplification allows us to determine the non-trivial infrared fixed point: the $s u(2)$ level 0 degrees of freedom decouple in the flow and the infrared conformal field theory is simply the coset $\operatorname{osp}(4 \mid 4)_{1} / s u(2)_{0}$. In this way we recover some of the percolation exponents predicted in [4].

Due to the logarithmic nature of the above conformal field theories, we find that in spite of the separation of the stress tensor into commuting pieces, $T_{o s p(4 \mid 4)}=T_{o s p(2 \mid 2)}+$ $T_{s u(2)}$, the Hilbert space does not factorize. This leads to the peculiar result that the coset $\operatorname{osp}(4 \mid 4)_{1} / \operatorname{su}(2)_{0}$ is not equivalent to the $\operatorname{osp}(2 \mid 2)_{-2}$ current algebra theory, even though this coset possesses the current algebra as a symmetry and the conformal dimensions of the coset are the same as for the $\operatorname{osp}(2 \mid 2)_{-2}$ current algebra.

In the last section we study the possible universality classes based on the 1-loop RG equations and suggest that the network model is universally attracted to a so-called "strange direction". 


\section{THE MODELS}

Kagalovsky et. al. gave a hamiltonian formulation of their network model [1]. The result is the $4 \times 4$ matrix hamiltonian:

$$
H=\left(\tau_{x} p_{x}+\tau_{z} p_{y}\right) \otimes 1+1 \otimes \vec{\alpha} \cdot \vec{\sigma}
$$

where $\vec{\tau}, \vec{\sigma}$ are two copies of the Pauli matrices, $p_{x, y}=-i \partial_{x, y}$, and $\vec{\alpha}$ is a random spin potential. This hamiltonian has the defining properties to belong the class $\mathrm{C}$ of the classification introduced in ref. [5]. Let us perform a unitary transformation $H \rightarrow\left(U^{\dagger} \otimes 1\right) H(U \otimes 1)$, where $U$ corresponds to a rotation about the $x$ axis for the Pauli matrices: $U^{\dagger} \tau_{z} U=\tau_{y}$, $U^{\dagger} \tau_{y} U=-\tau_{z}, U^{\dagger} \tau_{x} U=\tau_{x}$. After including $s u(2)$ gauge potentials to $\vec{p}$, one has the following $2 \times 2$ block structure:

$$
H=\left(\begin{array}{cc}
\vec{\alpha} \cdot \vec{\sigma} & -i \partial_{\bar{z}}+A_{\bar{z}} \\
-i \partial_{z}+A_{z} & \vec{\alpha} \cdot \vec{\sigma}
\end{array}\right)
$$

where $\partial_{z}=\partial_{x}-i \partial_{y}, \partial_{\bar{z}}=\partial_{x}+i \partial_{y}, A_{z}=A_{x}-i A_{y}, A_{\bar{z}}=A_{x}+i A_{y}$. In the above hamiltonian, $\vec{\alpha}(x, y)$ is a real, random spin potential, and $A_{\mu}=\sum_{a} A_{\mu}^{a}(x, y) \sigma^{a}$ are random $s u(2)$ gauge potentials, with $A_{x, y}^{a}$ real. In the sequel we will take them to have the following gaussian distributions

$$
\begin{aligned}
P(\vec{\alpha}) & =\exp \left(-\frac{1}{g_{\alpha}} \int \frac{d^{2} x}{2 \pi} \vec{\alpha}(x) \cdot \vec{\alpha}(x)\right) \\
P\left(A_{\mu}\right) & =\exp \left(-\frac{4}{g_{A}} \int \frac{d^{2} x}{2 \pi} A_{z}^{a}(x) A_{\bar{z}}^{a}(x)\right)
\end{aligned}
$$

As we will show in the next section, renormalization of the effective action obtained upon disorder averaging leads to an additional interaction which can be viewed as arising from a random mass $m(x, y)$. The complete hamiltonian which leads to a renormalizable effective action is then

$$
H=\left(\begin{array}{cc}
\vec{\alpha} \cdot \vec{\sigma}+m & -i \partial_{\bar{z}}+A_{\bar{z}} \\
-i \partial_{z}+A_{z} & \vec{\alpha} \cdot \vec{\sigma}-m
\end{array}\right)
$$

We will take $m(x)$ to have the gaussian distribution

$$
P(m)=\exp \left(-\frac{1}{g_{m}} \int \frac{d^{2} x}{2 \pi} m(x)^{2}\right)
$$

Note that since $\partial_{z}^{\dagger}=-\partial_{\bar{z}}, A_{z}^{\dagger}=A_{\bar{z}}$, the hamiltonian is hermitian if $m, \vec{\alpha}, A_{x, y}$ are real. In this situation the couplings $g_{\alpha}, g_{m}, g_{A}$ are the variances of normalizable gaussian distributions if they are real and positive. Negative couplings $g_{\alpha, m, A}$ can be interpreted as corresponding to imaginary random potentials; hermitian hamiltonians can then be constructed by doubling the number of degrees of freedom, as in [10].

The single particle Green functions are defined by the functional integral $Z^{-1} \int D \Psi^{*} D \Psi \exp (-S)$ with $Z$ the partition function and

$$
S=\int \frac{d^{2} x}{2 \pi} \Psi^{\star}(x) i(H-\mathcal{E}) \Psi(x)
$$


where $\mathcal{E}=E+i \varepsilon$. For $\varepsilon=0^{+}$, this defines the retarded Green function

$$
G_{R}\left(x, x^{\prime} ; E\right)=\lim _{\varepsilon \rightarrow 0^{+}}\left\langle x\left|\frac{1}{H-(E+i \varepsilon)}\right| x^{\prime}\right\rangle=\lim _{\varepsilon \rightarrow 0^{+}} i\left\langle\Psi(x) \Psi^{\star}\left(x^{\prime}\right)\right\rangle
$$

Letting

$$
\Psi=\left(\begin{array}{c}
\bar{\psi}_{+} \\
\psi_{+}
\end{array}\right), \Psi^{\star}=\left(\psi_{-}, \bar{\psi}_{-}\right)
$$

where $\psi_{+}$is a 2 -component fermion $\psi_{+}^{i}, i=1,2$, and similarly for $\bar{\psi}_{ \pm}$, one finds

$$
\begin{aligned}
S=\int \frac{d^{2} x}{2 \pi}\left(\psi_{-}\right. & \left(\partial_{\bar{z}}+i A_{\bar{z}}\right) \psi_{+}+\bar{\psi}_{-}\left(\partial_{z}+i A_{z}\right) \bar{\psi}_{+}+i \vec{\alpha} \cdot\left(\psi_{-} \vec{\sigma} \bar{\psi}_{+}+\bar{\psi}_{-} \vec{\sigma} \psi_{+}\right) \\
& \left.+i m\left(\psi_{-} \bar{\psi}_{+}-\bar{\psi}_{-} \psi_{+}\right)-i \mathcal{E} \Phi_{E}\right)
\end{aligned}
$$

where

$$
\Phi_{E}=\psi_{-} \bar{\psi}_{+}+\bar{\psi}_{-} \psi_{+}
$$

(We have suppressed the $s u(2)$ indices, i.e. $\psi_{-} \bar{\psi}_{+}=\sum_{i} \psi_{-}^{i} \bar{\psi}_{+}^{i}$, etc.)

In [1] the perturbation $H_{\epsilon}=\epsilon \tau_{y} \otimes 1$ was added and critical exponents for $\epsilon$ were measured numerically. Performing the unitary transformation $U$ defined above, this results in the following perturbation of the action:

$$
S_{\epsilon}=-i \epsilon \int \frac{d^{2} x}{2 \pi} \Phi_{\epsilon}, \quad \Phi_{\epsilon}=\psi_{-} \bar{\psi}_{+}-\bar{\psi}_{-} \psi_{+}
$$

Non-zero $\epsilon$ corresponds to a non-zero average random mass $m$.

Let us attempt to compare this with the model considered by Senthil et. al. [3]. There, one had a one-dimensional lattice in the $x$-direction with sites labeled by $j$, and a continuous $y$ direction. At each site there are fermionic degrees of freedom $\chi_{j}(y)$, where $j$ even corresponds to left-movers and $j$ odd to right-movers. The hamiltonian is

$$
\begin{gathered}
H=\sum_{j} \int d y\left((-)^{j} \chi_{j}^{\dagger}(y)\left(-i \partial_{y}+\vec{\eta}_{j}(y) \cdot \vec{\sigma}\right) \chi_{j}(y)-i t_{j}^{0}(y)\left(\chi_{j+1}^{\dagger} \chi_{j}-\chi_{j}^{\dagger} \chi_{j+1}\right)\right. \\
\left.+\vec{t}_{j}(y) \cdot\left(\chi_{j+1}^{\dagger} \vec{\sigma} \chi_{j}+\chi_{j}^{\dagger} \vec{\sigma} \chi_{j+1}\right)\right)
\end{gathered}
$$

Taking a continuum limit, $\chi_{j}(y) \rightarrow \psi_{+}(x, y)$ for $j$ even and $\chi_{j}(y) \rightarrow \bar{\psi}_{+}(x, y)$ for $j$ odd, $\vec{\eta}_{j}(y) \rightarrow \vec{\eta}_{y}(x, y)$, and $t_{j}(y) \rightarrow t(x, y)$, one finds

$$
\begin{gathered}
i H=\int d x d y\left(\psi_{-}\left(\partial_{y}+i \vec{\eta}_{y} \cdot \vec{\sigma}\right) \psi_{+}-\bar{\psi}_{-}\left(\partial_{y}+i \vec{\eta}_{y} \cdot \vec{\sigma}\right) \bar{\psi}_{+}+t_{0}\left(\psi_{-} \bar{\psi}_{+}-\bar{\psi}_{-} \psi_{+}\right)\right. \\
\left.+i \vec{t} \cdot\left(\psi_{-} \vec{\sigma} \bar{\psi}_{+}+\bar{\psi}_{-} \vec{\sigma} \psi_{+}\right)\right)
\end{gathered}
$$

Rotational invariance of the kinetic terms can be restored by adding $\partial_{x}+i \vec{\eta}_{x} \cdot \vec{\sigma}$ to the derivatives. Performing a rotation to euclidean space $y \rightarrow-i y$, one then obtains the action (2.9) with $\vec{\alpha}=\vec{t}, A_{z}=\left(\vec{\eta}_{x}-i \vec{\eta}_{y}\right) \cdot \vec{\sigma}$ and $t_{0}=i m$.

In [3], $\vec{t}$ and $t_{0}$ were taken as real gaussian distributed but with the same variance. For the model defined by (2.9), this corresponds to imaginary $m$. Letting $m \rightarrow-i m$, one sees 
that for the effective theory obtained after disorder averaging, imaginary $m$ corresponds to negative $g_{m}$. Since the variances are the same, this corresponds to $g_{\alpha}=-g_{m}$. I In summary, though we have not described an exact mapping between the network model in [1] and the super spin chain in [3, [1], it appears that they should be related on the line $g_{\alpha}+g_{m}=0$. In fact, one obtains directly the Heisenberg type superspin chain upon disorder averaging only along this line [3]. We will provide further support of this statement based on symmetry in the sequel. As we will show, this line has some rather special properties which allow the model to be solved.

\section{EFFECTIVE ACTION AND RENORMALIZATION GROUP ANALYSIS}

\section{A. Supersymmetric Disorder Averaging}

Since we are dealing with a free field theory, the supersymmetric method for disorder averaging can be used. Conformal field theory techniques in conjunction with this method was used for other models in [6] [7]. We augment the theory with bosonic ghosts $\beta_{ \pm}^{i}, \bar{\beta}_{ \pm}^{i}$, $i=1,2$, so that the inverse of the fermionic partition function $Z(\vec{\alpha}, m, A)=\int D \psi e^{-S(\psi)}$ is represented as a bosonic functional integral:

$$
Z(\vec{\alpha}, m, A)^{-1}=\int D \beta e^{-S(\psi \rightarrow \beta)}
$$

One can then perform the gaussian integrals over the random potentials. The result is the effective action:

$$
S_{e f f}=S_{c f t}+\int \frac{d^{2} x}{2 \pi}\left(g_{\alpha} \mathcal{O}_{\alpha}+g_{m} \mathcal{O}_{m}+g_{A} \mathcal{O}_{A}\right)
$$

The conformal field theory $S_{c f t}$ has Virasoro central charge $c=0$ and the action:

$$
S_{c f t}=\int \frac{d^{2} x}{2 \pi}\left(\psi_{-} \partial_{\bar{z}} \psi_{+}+\bar{\psi}_{-} \partial_{z} \bar{\psi}_{+}+\beta_{-} \partial_{\bar{z}} \beta_{+}+\bar{\beta}_{-} \partial_{z} \bar{\beta}_{+}\right)
$$

The first-order action for the bosonic ghosts of conformal dimension $\pm 1 / 2$ can be treated as in 88. The operators which perturb away from the conformal field theory are:

$$
\begin{aligned}
\mathcal{O}_{\alpha} & =\frac{1}{4}\left(\psi_{-} \vec{\sigma} \bar{\psi}_{+}+\bar{\psi}_{-} \vec{\sigma} \psi_{+}+\beta_{-} \vec{\sigma} \bar{\beta}_{+}+\bar{\beta}_{-} \vec{\sigma} \beta_{+}\right)^{2} \\
\mathcal{O}_{m} & =\frac{1}{4}\left(\psi_{-} \bar{\psi}_{+}-\bar{\psi}_{-} \psi_{+}+\beta_{-} \bar{\beta}_{+}-\bar{\beta}_{-} \beta_{+}\right)^{2} \\
\mathcal{O}_{A} & =\frac{1}{4}\left(\psi_{-} \vec{\sigma} \psi_{+}+\beta_{-} \vec{\sigma} \beta_{+}\right) \cdot\left(\bar{\psi}_{-} \vec{\sigma} \bar{\psi}_{+}+\bar{\beta}_{-} \vec{\sigma} \bar{\beta}_{+}\right)
\end{aligned}
$$

\footnotetext{
${ }^{1}$ This appears to be inconsistent with hermiticity, since the hamiltonian (2.4) is not hermitian for imaginary $m$, suggesting the two models cannot be naively compared in this way.
} 


\section{B. Beta Functions}

The one-loop renormalization group $\beta$ eta functions can be deduced from the operator product expansions of the marginal perturbing operators $\mathcal{O}_{i}$ [9]. Namely, if

$$
\mathcal{O}_{i}(z, \bar{z}) \mathcal{O}_{j}(0) \sim \frac{1}{z \bar{z}} C_{i j}^{k} \mathcal{O}_{k}(0)+\ldots
$$

then to lowest order the $\beta$ eta function $\beta_{k}=d g_{k} / d \log l$, where $l$ is a length scale, is given by

$$
\beta_{k}=-\sum_{i, j} C_{i j}^{k} g_{i} g_{j}
$$

We normalize the Pauli matrices as follows: $\vec{\sigma} \cdot \vec{\sigma}=\left(\sigma^{3}\right)^{2}+\sigma^{+} \sigma^{-}+\sigma^{-} \sigma^{+}$, with

$$
\sigma^{3}=\left(\begin{array}{cc}
1 & 0 \\
0 & -1
\end{array}\right), \quad \sigma^{+}=\sqrt{2}\left(\begin{array}{cc}
0 & 1 \\
0 & 0
\end{array}\right), \quad \sigma^{-}=\sqrt{2}\left(\begin{array}{cc}
0 & 0 \\
1 & 0
\end{array}\right)
$$

With this normalization, one has $\operatorname{Tr} \sigma^{a} \sigma^{b}=2 \delta^{a b}$, and $f^{a b c} f^{a b d}=-8 \delta^{c d}$, where $\left[\sigma^{a}, \sigma^{b}\right]=$ $f^{a b c} \sigma^{c}$. One also needs the operator products:

$$
\begin{aligned}
& \psi_{+}(z) \psi_{-}(0) \sim \psi_{-}(z) \psi_{+}(0) \sim 1 / z \\
& \beta_{+}(z) \beta_{-}(0) \sim-\beta_{-}(z) \beta_{+}(0) \sim 1 / z
\end{aligned}
$$

and similarly for the right-movers $\bar{\psi}, \bar{\beta}$ with $z$ replaced by $\bar{z}$. One then finds

$$
\begin{aligned}
\mathcal{O}_{A}(z, \bar{z}) \mathcal{O}_{A}(0) & \sim \frac{-2}{z \bar{z}} \mathcal{O}_{A}, \quad \mathcal{O}_{m}(z, \bar{z}) \mathcal{O}_{m}(0) \sim \frac{2}{z \bar{z}} \mathcal{O}_{m} \\
\mathcal{O}_{\alpha}(z, \bar{z}) \mathcal{O}_{\alpha}(0) & \sim \frac{1}{z \bar{z}}\left(2 \mathcal{O}_{\alpha}-8 \mathcal{O}_{A}\right) \\
\mathcal{O}_{A}(z, \bar{z}) \mathcal{O}_{\alpha}(0) & \sim \frac{1}{z \bar{z}}\left(-\frac{1}{2} \mathcal{O}_{\alpha}-\frac{3}{2} \mathcal{O}_{m}\right) \\
\mathcal{O}_{A}(z, \bar{z}) \mathcal{O}_{m}(0) & \sim \frac{1}{z \bar{z}}\left(-\frac{3}{2} \mathcal{O}_{m}-\frac{1}{2} \mathcal{O}_{\alpha}\right) \\
\mathcal{O}_{\alpha}(z, \bar{z}) \mathcal{O}_{m}(0) & \sim \frac{1}{z \bar{z}}\left(3 \mathcal{O}_{m}-\mathcal{O}_{\alpha}-4 \mathcal{O}_{A}\right)
\end{aligned}
$$

The resulting $\beta$ eta functions are

$$
\begin{aligned}
& \beta_{\alpha}=-2 g_{\alpha}^{2}+2 g_{\alpha} g_{m}+g_{A}\left(g_{\alpha}+g_{m}\right) \\
& \beta_{m}=-2 g_{m}^{2}-6 g_{\alpha} g_{m}+3 g_{A}\left(g_{\alpha}+g_{m}\right) \\
& \beta_{A}=2 g_{A}^{2}+8 g_{\alpha}\left(g_{\alpha}+g_{m}\right)
\end{aligned}
$$

If one starts with a model with only the random spin potential $\vec{\alpha}$, i.e. only $g_{\alpha} \neq 0$, then $g_{A}$ and $g_{m}$ are generated under renormalization. Note that the $\beta$ eta functions simplify dramatically when $g_{\alpha}+g_{m}=0$, which is the subject of the next section. We will return to a general analysis of the possible universality classes contained in these $\beta$ eta functions in section $\mathrm{V}$. 


\section{SPIN-CHARGE SEPARATION}

\section{A. Symmetries of the effective action}

The free conformal action $S_{c f t}$ has a maximal $\operatorname{osp}(4 \mid 4)_{1}$ current algebra symmetry. The conformal currents $J^{A}$ generating this symmetry correspond to all possible bilinears in fermions and ghosts. Suppressing the possible index structure, $J^{A}=\{\psi \psi, \psi \beta, \beta \beta\}$. The perturbing operators are bilinear in these currents: $\mathcal{O}_{\alpha}=d_{A B}^{(\alpha)} J^{A} \bar{J}^{B}$ for some tensor $d_{A B}^{(\alpha)}$ and similarly for $\mathcal{O}_{m}$ and $\mathcal{O}_{A}$.

For arbitrary $g_{\alpha}, g_{m}, g_{A}$, the $\operatorname{osp}(4 \mid 4)$ symmetry of $S_{c f t}$ is broken but an $\operatorname{osp}(2 \mid 2)$ symmetry is preserved. To describe the symmetry, let us define the left-moving currents:

$$
\begin{array}{rlrl}
J & =\sum_{i} \psi_{+}^{i} \psi_{-}^{i}, & J_{ \pm} & =\sum_{i, j} \epsilon_{i j} \psi_{ \pm}^{i} \psi_{ \pm}^{j} \\
H & =\sum_{i} \beta_{+}^{i} \beta_{-}^{i}, & S_{ \pm}=\sum_{i} \psi_{ \pm}^{i} \beta_{\mp}^{i} \\
\widehat{S}_{ \pm} & =\sum_{i, j} \epsilon_{i j} \psi_{ \pm}^{i} \beta_{ \pm}^{j} & &
\end{array}
$$

where $\epsilon_{i j}=-\epsilon_{j i}, \epsilon_{12}=1$, and similarly for the right-movers $\bar{J}=\bar{\psi}_{+} \bar{\psi}_{-}, \bar{H}=\bar{\beta}_{+} \bar{\beta}_{-}, \ldots \ldots$ These currents generate an $\operatorname{ssp}(2 \mid 2)_{k}$ current algebra at level $k=-2$. The non-zero operator products are

$$
\begin{array}{rlrl}
J(z) J(0) & \sim-\frac{k}{z^{2}}, & H(z) H(0) & \sim \frac{k}{z^{2}} \\
J(z) J_{ \pm}(0) & \sim \pm \frac{2}{z} J_{ \pm}, & J_{+}(z) J_{-}(0) & \sim \frac{2 k}{z^{2}}-\frac{4}{z} J \\
J(z) S_{ \pm}(0) & \sim \pm \frac{1}{z} S_{ \pm}, & J(z) \widehat{S}_{ \pm}(0) & \sim \pm \frac{1}{z} \widehat{S}_{ \pm} \\
H(z) S_{ \pm}(0) & \sim \pm \frac{1}{z} S_{ \pm}, & H(z) \widehat{S}_{ \pm}(0) & \sim \mp \frac{1}{z} \widehat{S}_{ \pm} \\
J_{ \pm}(z) S_{\mp}(0) & \sim \frac{2}{z} \widehat{S}_{ \pm}, & J_{ \pm}(z) \widehat{S}_{\mp}(0) & \sim-\frac{2}{z} S_{ \pm} \\
S_{ \pm}(z) \widehat{S}_{ \pm}(0) & \sim \pm \frac{1}{z} J_{ \pm} & & \\
S_{+}(z) S_{-}(0) & \sim \frac{k}{z^{2}}+\frac{1}{z}(H-J) & \\
\widehat{S}_{+}(z) \widehat{S}_{-}(0) & \sim-\frac{k}{z^{2}}+\frac{1}{z}(H+J)
\end{array}
$$

The currents $J$ and $J_{ \pm}$generate a charged $s u(2)$ subalgebra under which the fermions $\psi_{ \pm}^{i}$ transform as doublets.

The $\operatorname{osp}(2 \mid 2)$ symmetry is present for arbitrary potentials before disorder averaging, as we now describe. The fermionic generators define nilpotent transformations of the bosonic and fermionic fields. The action (2.9) is then an exact variation with respect to these transformations, making then clear the fact that this $\operatorname{osp}(2 \mid 2)$ symmetry is preserved. For example the generator $S_{+}$induces the transformation $\delta \psi_{+}^{i}=0, \delta \psi_{-}^{i}=\beta_{-}^{i}, \delta \beta_{+}^{i}=-\psi_{+}^{i}$, 
$\delta \beta_{-}^{i}=0$. The symmetry acts left-right diagonally, so for the right movers $\delta \bar{\psi}_{+}^{i}=0, \delta \bar{\psi}_{-}^{i}=$ $\bar{\beta}_{-}^{i}, \delta \bar{\beta}_{+}^{i}=-\bar{\psi}_{+}^{i}, \delta \bar{\beta}_{-}^{i}=0$. The action (2.9) may be written as

$$
S=S_{c f t}+\delta \int \frac{d^{2} x}{2 \pi} \Theta
$$

where

$$
\Theta=i m\left(\psi_{-} \bar{\beta}_{+}-\bar{\psi}_{-} \beta_{+}\right)+i \vec{\alpha} \cdot\left(\psi_{-} \vec{\sigma} \bar{\beta}_{+}+\bar{\psi}_{-} \vec{\sigma} \beta_{+}\right)+i \vec{A} \cdot\left(\psi_{-} \vec{\sigma} \beta_{+}+\bar{\psi}_{-} \vec{\sigma} \bar{\beta}_{+}\right)
$$

Hence $\delta S=0$ since $\delta S_{c f t}=0$ and $\delta^{2}=0$. This holds similarly, but with another operator $\widehat{\Theta}$, for $\widehat{S}_{-}$which generates the transformations $\widehat{\delta} \psi_{+}^{i}=\epsilon^{i j} \beta_{-}^{j}, \widehat{\delta} \psi_{-}^{i}=0$ and $\widehat{\delta} \beta_{+}^{i}=\epsilon^{i j} \psi_{-}^{j}$, $\widehat{\delta} \beta_{-}^{i}=0$. This $\operatorname{osp}(2 \mid 2)$ symmetry would not be preserved if we had chosen the random gauge potential in $u(2)$ or if had added extra scalar potential randomness.

This implies that after disorder averaging the perturbations by $\mathcal{O}_{\alpha}, \mathcal{O}_{m}$ and $\mathcal{O}_{A}$ in the effective theory preserve a global, left-right diagonal, $\operatorname{osp}(2 \mid 2)$ symmetry. The current conservation law takes the left-right diagonal form

$$
\partial_{\bar{z}} J^{a}+\partial_{z} \bar{J}^{a}=0
$$

for $J^{a}, \bar{J}^{a}$ any of the eight $\operatorname{osp}(2 \mid 2)_{-2}$ currents.

Let us describe more explicitly how the $\operatorname{osp}(2 \mid 2)$ symmetry is manifested in the effective theory. In the sequel we will set $g_{\alpha}=-g_{m}=g$ and the effective action will contain the operator $\mathcal{O}_{g} \equiv \mathcal{O}_{\alpha}-\mathcal{O}_{m}$. Many terms cancel in the combination $\mathcal{O}_{\alpha}-\mathcal{O}_{m}$ and the result can be written as an $\operatorname{ssp}(2 \mid 2)$ current-current perturbation. This simplification is analogous to the $g_{V}+g_{M}=0$ line for the random $U(1)$ fermions which has $g l(1 \mid 1)$ symmetry, studied in [10]. By repeated use of the identity

$$
\sigma_{i j}^{a} \sigma_{n m}^{a}=2 \delta_{i m} \delta_{j n}-\delta_{i j} \delta_{n m}
$$

one can express $\mathcal{O}_{g}$ in terms of the above $\operatorname{osp}(2 \mid 2)$ currents:

$$
\begin{aligned}
\mathcal{O}_{g} & \equiv \mathcal{O}_{\alpha}-\mathcal{O}_{m} \\
& =-J \bar{J}+H \bar{H}+\frac{1}{2}\left(J_{-} \bar{J}_{+}+J_{+} \bar{J}_{-}\right)+S_{-} \bar{S}_{+}-S_{+} \bar{S}_{-}-\widehat{S}_{-} \overline{\widehat{S}}_{+}+\widehat{S}_{+} \overline{\widehat{S}}_{-}
\end{aligned}
$$

The operator $\mathcal{O}_{g}$ has the structure of the quadratic Casimir for $\operatorname{osp}(2 \mid 2)$. Namely, $\mathcal{O}_{g}=$ $\sum_{a, b} C_{a b} J^{a} \bar{J}^{b}$, where $J^{a}$ are $\operatorname{osp}(2 \mid 2)$ currents and $C_{a b}$ corresponds to the quadratic Casimir. Thus $\mathcal{O}_{g}$ is $\operatorname{osp}(2 \mid 2)$ invariant.

The operator $\mathcal{O}_{\alpha}+\mathcal{O}_{m}$ cannot be written only in terms of the $\operatorname{osp}(2 \mid 2)$ currents; one needs some of the $\operatorname{ssp}(4 \mid 4)$ currents. Let

$$
\begin{aligned}
& B_{ \pm}^{i j}=\beta_{ \pm}^{i} \beta_{ \pm}^{j} \quad ; \quad \vec{L}_{f}=\psi_{-} \vec{\sigma} \psi_{+} \quad ; \quad \vec{L}_{b}=\beta_{-} \vec{\sigma} \beta_{+} \\
& U_{ \pm}^{i j}=\frac{1}{2}\left(\beta_{ \pm}^{i} \psi_{ \pm}^{j}+\beta_{ \pm}^{j} \psi_{ \pm}^{i}\right) \quad ; \quad \vec{V}_{-}=\psi_{-} \vec{\sigma} \beta_{+} \quad ; \quad \vec{V}_{+}=\beta_{-} \vec{\sigma} \psi_{+}
\end{aligned}
$$

Then,

$$
\mathcal{O}_{\alpha}+\mathcal{O}_{m}=\frac{1}{2} \mathcal{O}_{g}+\widetilde{\mathcal{O}}
$$


with

$$
\begin{aligned}
\widetilde{\mathcal{O}}= & \frac{1}{2}\left(B_{-}^{i j} \bar{B}_{+}^{i j}+B_{+}^{i j} \bar{B}_{-}^{i j}\right)+\frac{1}{2}\left(\vec{L}_{f} \cdot \vec{L}_{f}-\vec{L}_{b} \cdot \overrightarrow{\bar{L}}_{b}\right) \\
& +U_{-}^{i j} \bar{U}_{+}^{i j}-U_{+}^{i j} \bar{U}_{-}^{i j}+\frac{1}{2}\left(\vec{V}_{+} \cdot \vec{V}_{-}-\vec{V}_{-} \cdot \vec{V}_{+}\right)
\end{aligned}
$$

We have already seen that $\mathcal{O}_{g}$ is $\operatorname{osp}(2 \mid 2)$ invariant. One can check explicitly that $\widetilde{\mathcal{O}}$ is also $\operatorname{osp}(2 \mid 2)$ invariant.

Let us turn now to the perturbation $\mathcal{O}_{A}$. This operator as defined is a left-right currentcurrent perturbation:

$$
\mathcal{O}_{A}=\sum_{a=1}^{3} L^{a} \bar{L}^{a}
$$

where

$$
L^{a}=L_{f}^{a}+L_{b}^{a}=\psi_{-} \sigma^{a} \psi_{+}+\beta_{-} \sigma^{a} \beta_{+}
$$

The central extension (level) cancels between the fermions and ghosts and the result is that $L^{a}$ generate an $s u(2)_{k}$ current algebra at level $k=0$ :

$$
L^{a}(z) L^{b}(0) \sim \frac{1}{z} f^{a b c} L^{c}(0)+\operatorname{reg} .
$$

Again, since $\mathcal{O}_{A}$ takes the form of the Casimir of $s u(2), \mathcal{O}_{A}$ preserves a global su(2) symmetry. Furthermore it is easy to check that this global $s u(2)$ commutes with the $\operatorname{ssp}(2 \mid 2)$ symmetry:

$$
\left[L^{a}, J^{b}\right]=0
$$

where $J^{a}$ are the $\operatorname{osp}(2 \mid 2)$ currents defined in eq. (4.1).

In summary, the model has a global $\operatorname{osp}(2 \mid 2) \otimes s u(2)$ symmetry for general $g_{\alpha}, g_{m}, g_{A}$.

\section{B. Quasi-spin-charge separation in the conformal field theory}

Since $\mathcal{O}_{g}$ only involves the $\operatorname{osp}(2 \mid 2)_{-2}$ currents and $\mathcal{O}_{A}$ the $s u(2)_{0}$ currents, it is important to understand how the conformal field theory decomposes in terms of these current algebras. It is straightforward to check that the $s u(2)_{0}$ and $\operatorname{osp}(2 \mid 2)_{-2}$ current algebras commute, so that the conformal field theory defined by $S_{c f t}$ in eq. (3.3) has an $\operatorname{sep}(2 \mid 2)_{-2} \otimes s u(2)_{0}$ symmetry.

We now show the much stronger result that the full stress tensor, which is the Sugawara stress tensor for the $\operatorname{ssp}(4 \mid 4)_{1}$ supercurrent algebra, separates into two commuting pieces:

$$
T_{o s p(4 \mid 4)_{1}}=T_{o s p(2 \mid 2)_{-2}}+T_{s u(2)_{0}}
$$

All T's in the above equation are Sugawara stress tensors and have Virasoro central charge equal to zero. The above equation is proven in the appendix.

Since $\operatorname{ssp}(2 \mid 2)_{-2}$ contains charged currents whereas $s u(2)_{0}$ does not, the equation (4.12) implies a kind of spin-charge separation and we will use this terminology in the sequel. 
Based on the separation (4.12) one would expect that the Hilbert space of $\operatorname{sep}(4 \mid 4)_{1}$ factorizes. However by trying to perform explicitly this factorization in some simple cases, we found that it is not possible:

$$
\mathcal{H}_{o s p(4 \mid 4)_{1}} \neq \mathcal{H}_{o s p(2 \mid 2)_{-2}} \otimes \mathcal{H}_{s u(2)_{0}}
$$

This non-factorization is intimitely related to the way the fields that appear in $\widetilde{\mathcal{O}}$ transform under $\operatorname{ssp}(2 \mid 2)$. This is described in Figure 2. In particular, the $s u(2)_{0}$ currents $L^{a}$ turn out to be susy exact, e.g.:

$$
S_{+}(z) V_{-}^{a}(0) \sim \frac{1}{z} L^{a}
$$

Let us argue for the non-factorazibility of the Hilbert space by contradiction. The 24 fields of $\widetilde{\mathcal{O}}$ form three copies of an eight dimensional reducible but indecomposable representation of $\operatorname{osp}(2 \mid 2)$, that we shall denote $[\widetilde{8}]$. Consider the state $\left|L_{A}^{a}\right\rangle \equiv\left(L_{f,-1}^{a}-L_{b,-1}^{a}\right)|0\rangle$, where $L_{-1}^{a}=\oint d z L^{a}(z) / 2 i \pi z$. If the factorization of $\mathcal{H}_{o s p(4 \mid 4)_{1}}$ holds, then

$$
\left|L_{A}^{a}\right\rangle=\sum_{i}|o s p\rangle_{i} \otimes|s u\rangle_{i} \in[\widetilde{8}] \otimes[3]
$$

where [3] is the adjoint representation of $s u(2)$. Let us now act on $\left|L_{A}^{a}\right\rangle$ with the $s u(2)_{0}$ current. As a state in $\mathcal{H}_{o s p(4 \mid 4)_{1}}$ one finds that $L_{-1}^{b}\left|L_{A}^{a}\right\rangle=\delta^{a b}\left|0_{\text {Fock }}\right\rangle$ with $\left|0_{\text {Fock }}\right\rangle$ the vacuum of the Fock space. As a state in $\mathcal{H}_{o s p(2 \mid 2)_{-2}} \otimes \mathcal{H}_{s u(2)_{0}}$, one would find

$$
L_{-1}^{b}\left|L_{A}^{a}\right\rangle=\sum|o s p\rangle_{i} \otimes L_{-1}^{b}|s u\rangle_{i} \in[\tilde{8}] \otimes[1]
$$

where [1] is the $s u(2)$ singlet. Thus if the factorization holds, one would deduce that

$$
\left|0_{\text {Fock }}\right\rangle \in[\widetilde{8}] \otimes[1]
$$

Since $\left|0_{\text {Fock }}\right\rangle$ is annihilated by all $\operatorname{osp}(2 \mid 2)$ generators, this would mean that $\left|0_{\text {Fock }}\right\rangle$ is in the image of susy generators, i.e. there would exist a state $|\Omega\rangle$ such that for example $\left|0_{\text {Fock }}\right\rangle=S_{-}|\Omega\rangle$. This is clearly a contradiction. A similar argument shows that eq. (4.13) leads to a contradiction in the factorization of $V_{-}^{a}$.

Another argument for the non-factorization is based on the fact that the current algebras $\operatorname{osp}(2 \mid 2)_{-2}$ and $\operatorname{su}(2)_{0}$ lead to logarithmic correlation functions [13] [14], whereas the $o s p(4 \mid 4)_{1}$ is a free theory with no logarithms. It appears impossible for these logarithms to cancel in the product of two logarithmic correlation functions. The logarithmic nature of these theories can in fact be traced to transformations such as (4.13) [13].

We now describe a few of the results we need concerning these current algebra conformal field theories. Representations of $\operatorname{ssp}(2 \mid 2)$ are characterized by the quantum numbers of the $s u(2) \otimes u(1)$ subalgebra generated by $\left(J, J_{ \pm}\right)$and $H$. Highest weights can be labeled $(j, b)$ where $j=0,1 / 2,1, .$. is the spin of the charge-su(2) and $b=H / 2$. These representations have dimension $8 j$. The conformal scaling dimension (left or right-moving) of the corresponding primary fields, as determined from the Sugarawa construction, is [12,13]

$$
\Delta_{(j, b)}^{o s p(2 \mid 2)}=\frac{2\left(j^{2}-b^{2}\right)}{2-k}
$$


On the other hand, the primary fields of the $s u(2)_{k}$ current algebra are characterized by the spin $j$ of the spin-su(2) only and have conformal dimension [11]

$$
\Delta_{j}^{s u(2)}=\frac{j(j+1)}{k+2}
$$

A simple check of eq. (4.12) is based on the dimensions of the eight original fermion and ghost fields $\psi_{ \pm}^{i}, \beta_{ \pm}^{i}$. In the original conformal field theory these fields have conformal dimension $\Delta=1 / 2$. Under the $\operatorname{osp}(2 \mid 2)$ they transform according to the two four-dimensional representations $\left(\psi_{+}^{1}, \psi_{-}^{2}, \beta_{-}^{2}, \beta_{+}^{1}\right)$ and $\left(\psi_{-}^{1}, \psi_{+}^{2}, \beta_{+}^{2}, \beta_{-}^{1}\right)$ corresponding to $(j=1 / 2, b=0)$ and its conjugate. At level $k=-2$, these have dimension $\Delta^{o s p(2 \mid 2)}=1 / 8$. The same fields transform as spin $j=1 / 2$ doublets according to the $s u(2)_{0}$, and have dimension $\Delta^{s u(2)}=3 / 8$ at level zero. The exact decomposition (4.12) implies that these dimensions must add up properly: $1 / 2=1 / 8+3 / 8$.

\section{Infrared fixed points and critical exponents}

We now study the model when $g_{\alpha}+g_{m}=0$. Setting $g_{\alpha}=-g_{m}=g$, the effective action contains the current-current operators $\mathcal{O}_{g}$ and $\mathcal{O}_{A}$. The operator $\widetilde{\mathcal{O}}$ which couples the $\operatorname{osp}(2 \mid 2)_{-2}$ and $s u(2)_{0}$ current algebras is not present. The model in [3] was mapped onto a super-spin chain with $\operatorname{ssp}(2 \mid 2)$ symmetry and Heisenberg-type hamiltonian. This further supports the identification of this super spin chain with our model on the line $g_{\alpha}+$

$g_{m}=0$, since, at least for ordinary bosonic algebras, Heisenberg hamiltonians correspond to symmetry preserving current-current perturbations built on the quadratic casimir in the continuum limit. (As Lie superalgebras, $\operatorname{osp}(2 \mid 2)$ and $s l(2 \mid 1)$ are identical.)

The effective action now contains interactions that do not couple the spin and charge currents:

$$
S_{e f f}=S_{\mathrm{cft}}+\int \frac{d^{2} x}{2 \pi}\left(g \mathcal{O}_{g}+g_{A} \mathcal{O}_{A}\right)
$$

This decoupling is of course consistent with the more general $\beta$ eta functions (3.10). Setting $g_{\alpha}=-g_{m}=g$ one finds

$$
\beta_{g}=-4 g^{2}, \quad \beta_{g_{A}}=2 g_{A}^{2}
$$

As explained in section II, the model of Senthil et. al. corresponds to $g$ and $g_{A}$ positive. From the above $\beta$ eta functions one sees that $g_{A}$ is marginally relevant whereas $g$ is marginally irrelevant. In order to deduce something exact concerning the infrared (IR) fixed point theory from the one-loop $\beta$ eta functions, one needs to make a hypothesis concerning the role of the higher loop corrections. Consider for comparison the $s u(N)$ Gross-Neveu models, which are $s u(N)$ current-current perturbations, with $\beta$ eta functions as in eq. (4.17), i.e. $\beta_{g}=g^{2}$ in a certain convention. When $g>0$ the perturbation is marginally relevant, i.e. $g$ grows at large distances. Higher loop corrections do not modify this, i.e. the flow to the IR does not stop at some finite value of $g$ corresponding to a non-trivial fixed point. Rather, $g$ eventually flows to infinity. The theory is thus massive, and in the infrared all these massive modes disappear leaving no massless degrees of freedom, i.e. the infrared theory is an empty theory. 
When $g<0$, the perturbation is marginally irrelevant, i.e. $g$ flows back to zero and the unperturbed conformal current algebra is recovered in the IR. We will make the hypothesis that this is the only possible behavior for general current-current perturbations, i.e. the only possible fixed points are $g=0$ or $\infty$.

For our model we then have the following picture. In the IR, $g_{A}$ flows to infinity and the spin degrees of freedom are massive and decouple. The coupling $g$ on the other hand is marginally irrelevant. Therefore the IR fixed point is the coset $\operatorname{osp}(4 \mid 4)_{1} / s u(2)_{0}$, and the theory arrives in the IR via the operator $\mathcal{O}_{g}$. Due to the non-factorizability of the Hilbert space, this coset conformal field theory is not precisely the $\operatorname{osp}(2 \mid 2)_{-2}$ current algebra, even though it possesses this current algebra as a symmetry and the conformal dimensions of the coset theory are the same as the $\operatorname{osp}(2 \mid 2)$ current algebra because of (4.12).

This identification of the IR fixed point allows the computation of certain critical exponents. The density of states $\rho(E)$ is

$$
\rho(E)=\frac{1}{V} \operatorname{Tr} \delta(H-E)=\frac{1}{\pi V} \lim _{\varepsilon \rightarrow 0^{+}} \operatorname{Im} \operatorname{Tr} \frac{1}{H-E-i \varepsilon}
$$

where $V$ is the two-dimensional volume. This implies that the disorder averaged density of states is proportional to the one-point correlation function:

$$
\overline{\rho(E)} \propto \operatorname{Re}\left\langle\Phi_{E}\right\rangle
$$

Let $\Gamma_{E}$ equal the scaling dimension of $\Phi_{E}$. Then, since the action (2.9) is dimensionless, viewing $E$ as a coupling, $\operatorname{dim}(E)=2-\Gamma_{E}$. Since $E$ is the only dimensionful coupling in the theory one deduces

$$
\overline{\rho(E)} \propto E^{\Gamma_{E} /\left(2-\Gamma_{E}\right)} \quad \text { as } \quad E \rightarrow 0
$$

One can also define a correlation length $\xi_{E}$,

$$
\xi_{E} \propto E^{-\nu_{E}}, \quad \nu_{E}=1 /\left(2-\Gamma_{E}\right)
$$

(In [3], $\nu_{E}$ was referred to as $\nu_{B}$.)

For our theory, $\Phi_{E}=\psi_{+} \bar{\psi}_{-}+\ldots$ and $\Gamma_{E}$ is the scaling dimension in the IR, which follows from the $\operatorname{osp}(2 \mid 2)_{-2}$ conformal dimension of $\psi_{ \pm}$, which as explained above is $\Delta_{(1 / 2,0)}^{o s p(2 \mid 2)}=1 / 8$. Thus $\Gamma_{E}=1 / 4$, and:

$$
\overline{\rho(E)} \propto E^{1 / 7}, \quad \nu_{E}=4 / 7
$$

Numerical simulations of the $\operatorname{osp}(2 \mid 2)$ invariant spin-chain agree very well with $\nu_{E}=4 / 7$ [3]. Since the system flows toward the infrared fixed point along a marginal direction, the scaling (4.22) is up to computable logarithmic corrections.

In the above scenario, the $s u(2)_{0}$ spin degrees of freedom are becoming localized, hence the terminology "spin quantum hall effect". The above model can also exhibit charge localization by changing the signs of the couplings. Namely, if $g$ and $g_{A}$ are negative, then $g$ is marginally relevant, i.e. it flows to $-\infty$ in the IR and $g_{A}$ is marginally irrelevant. Following the same reasoning as above, in this case the charge degrees of freedom decouple in the flow. The IR fixed point is now the coset $\operatorname{osp}(4 \mid 4)_{1} / \operatorname{osp}(2 \mid 2)_{-2}$. Here, $\Gamma_{E}=3 / 4$, and: 


$$
\overline{\rho(E)} \propto E^{3 / 5}, \quad \xi_{E} \propto E^{-4 / 5} \quad \text { for charge delocalization }
$$

Finally, if $g<0, g_{A}>0$, then $g, g_{A}$ flow to $-\infty$ and $\infty$ respectively, and both sectors are massive and decoupled in the IR. This implies $\Gamma_{E}=0$, and a constant density of states at $E=0$.

\section{Path Integral Factorization}

Let us now present a path integral derivation of the spin-charge separation which has the advantage of being non perturbative and thus valid all along the RG trajectory. One can view this formulation as a way of defining the coset $\operatorname{osp}(4 \mid 4)_{1} / s u(2)_{0}$. It consists in decoupling the random gauge field $A$ using chiral gauge transformations. The spin-charge separation will then be a consequence of the fact that the $\operatorname{ssp}(2 \mid 2)$ currents are invariant under the chiral gauge transformations. This decoupling is similar to the solution of the random gauge potential described in [6].

Let $S$ be the fermionic action (2.9) and $Z(A, \alpha, m)$ its partition function $Z(A, \alpha, m)=$ $\int D \Psi e^{-S(\Psi)}$. At a fixed realization of disorder, the gauge potential $A$ may be gauged away by a chiral gauge transformation by parameterizing $A$ as:

$$
i \bar{A}=G^{-1} \partial_{\bar{z}} G \quad ; \quad i A=G^{*} \partial_{z} G^{*-1}
$$

with $G$ an element of the complex $s u(2)^{C}$ group, i.e. $G$ is a two by two complex matrix with determinant one. This is always possible on the sphere. This parametization is such that $\psi_{-}\left(\partial_{\bar{z}}+i \bar{A}\right) \psi_{+}=\left(\psi_{-} G^{-1}\right) \partial_{\bar{z}}\left(G \psi_{+}\right)$. Let us now denote by $\psi^{\prime}$ the chiral gauge-transformed fermions:

$$
\begin{array}{rll}
\psi_{-}^{\prime}=\psi_{-} G^{-1} & ; \quad \bar{\psi}_{-}^{\prime}=\bar{\psi}_{-} G^{*} \\
\psi_{+}^{\prime}=G \psi_{+} \quad ; \quad \bar{\psi}_{+}^{\prime}=G^{*-1} \bar{\psi}_{+}
\end{array}
$$

Gauge transformed bosons $\beta^{\prime}$ are defined similarly. The fermionic action (2.9) may be rewritten in terms of $G$ and $\Psi^{\prime}$. It becomes:

$$
\begin{aligned}
\Gamma\left(\Psi^{\prime} \mid G, \alpha, m\right)=\int \frac{d^{2} x}{2 \pi} & \left(\psi_{-}^{\prime} \partial_{\bar{z}} \psi_{+}^{\prime}+\bar{\psi}_{-}^{\prime} \partial_{z} \bar{\psi}_{+}^{\prime}\right. \\
& \left.+i \psi_{-}^{\prime} G(\vec{\alpha} \cdot \vec{\sigma}-m) G^{*} \bar{\psi}_{+}^{\prime}+i \bar{\psi}_{-}^{\prime} G^{*-1}(\vec{\alpha} \cdot \vec{\sigma}+m) G^{-1} \psi_{+}^{\prime}\right)
\end{aligned}
$$

This is the effective action for the Dirac operator coupled to the disorder variables $G \vec{\alpha} \cdot \vec{\sigma} G^{*}$, $G^{*-1} \vec{\alpha} \cdot \vec{\sigma} G^{-1}$ and $G m G^{*}$. As we shall see below, the field $G$ will be coupled to the spin degrees of freedom whereas the fermions $\Psi^{\prime}$ shall be coupled to the charged sector. The density of states has a factorized expression in terms of these new spin and charged variables:

$$
\rho(E) \propto \operatorname{Re}\left\langle\bar{\Psi}^{\prime}\left(G G^{*}\right) \Psi^{\prime}\right\rangle
$$

We must not neglect to take into account the jacobians of the transformations $A \rightarrow G$ and $\Psi \rightarrow \Psi^{\prime}$ as well as to represent the partition function $Z(A, \alpha, m)$. The jacobians, which are determinants of Dirac operators, are computed using the chiral anomaly. They may be expressed with the help of the WZW action: 


$$
\begin{aligned}
\left|\frac{D \Psi}{D \Psi^{\prime}}\right| & =\operatorname{Det}(i \not \partial+A)=\exp \left(S_{w z w}\left(G G^{*}\right)\right) \\
\left|\frac{D A}{D G}\right| & =\exp \left(4 S_{w z w}\left(G G^{*}\right)\right)
\end{aligned}
$$

with $S_{w z w}$ the WZW action.

To represent the partition function, note that $\operatorname{Det}(i \not \partial+A)=Z(A, \alpha=0, m=0)$. Therefore, chiral gauge transformations applied to the bosonic beta system give:

$$
\frac{\operatorname{Det}(\not \not \supset+A)}{Z(A, \alpha, m)}=\int D \beta^{\prime} \exp \left(-\Gamma\left(\beta^{\prime} \mid G, \alpha, m\right)\right)
$$

Gathering the jacobians we get the action

$$
S=-4 S_{w z w}\left(G G^{*}\right)+\Gamma\left(\Psi^{\prime} \mid G, \alpha, m\right)+\Gamma\left(\beta^{\prime} \mid G, \alpha, m\right)
$$

with $\Gamma$ defined in eq.(4.26). This is the action to compute correlations using $G, \Psi^{\prime}$ and $\beta^{\prime}$ as the path integral variables at fixed disorder. One still has to add the disorder measure (2.3,2.5) to compute averaged correlations.

In eq.(4.29) the random spin variables $A$ or $G$ are not yet decoupled. This decoupling only appears when $g_{\alpha}+g_{m}=0$. Indeed, in that case integrating over the disorder $\alpha$ and $m$ yields to current-current type interactions as in eq.(4.6) but with $\operatorname{osp}(2 \mid 2)$ currents bilinear in the fermions $\Psi$ instead of the fermions $\Psi^{\prime}$. The crucial point is now to remark that these $\operatorname{osp}(2 \mid 2)$ currents are invariant under the chiral gauge transformations (4.25). For example,

$$
\begin{aligned}
\psi_{-}^{\prime} \cdot \psi_{+}^{\prime} & =\psi_{-} G G^{-1} \psi_{+}=\psi_{-} \cdot \psi_{+}=J \\
\epsilon_{i j} \psi_{+i}^{\prime} \psi_{+j}^{\prime} & =\psi_{+n} \psi_{+m} G_{i n} G_{j m} \epsilon_{i j}=\psi_{+n} \psi_{+m} \epsilon_{n m}=J^{-}
\end{aligned}
$$

where we used that $G_{i n} G_{j m} \epsilon_{i j}=\operatorname{det} G \epsilon_{n m}=\epsilon_{n m}$ since $G$ has unit determinant. Similarly, it is easily checked that all $\operatorname{ssp}(2 \mid 2)$ currents are invariant under chiral gauge transformations. Note that this is true because the spin disorder variables belong to $s u(2)$. This would not be valid if for example the spin disorder variables were taking values in $u(2)$ instead.

Hence, after integrating over the disorder at $g_{\alpha}+g_{m}=0$, the spin random variables $G$ decouple from the $\Psi^{\prime}$ and $\beta^{\prime}$ system. This is the spin-charge separation. We are thus left with the action:

$$
S=-4 S_{w z w}\left(G G^{*}\right)+\Gamma\left(\Psi^{\prime} \mid G=1, \alpha, m\right)+\Gamma\left(\beta^{\prime} \mid G=1, \alpha, m\right)
$$

with the same disorder measure for $G$ and $\alpha, m$ as in eq.(2.3.2.5) with $g_{A}$ arbitrary but $g_{\alpha}+g_{m}=0$. The first term describes a WZW theory on the coset space $s u(2)^{C} / s u(2)$ at level $k=-4$. This may be thought of as the theory 'inverse' to the $s u(2)$ WZW theory at level $k=0$ [15, since the conformal dimensions of primary field in the two theories have opposite sign. The second and third terms describe the $\operatorname{ssp}(2 \mid 2)$ current-current perturbation of the $\Psi^{\prime}-\beta^{\prime}$ system. As in previous section this $\Psi^{\prime}-\beta^{\prime}$ system may also be described as an $\operatorname{osp}(4 \mid 4)$ WZW model at level one and the current-current perturbation only couples to the sub-sector generated by the $\operatorname{osp}(2 \mid 2)$ currents.

In the last section we argued that $g_{A}$ flows to infinity under RG flow. At $g_{A}=\infty$, the measure (2.3) on $G$ is flat and only the WZW action $S_{w z w}\left(G G^{*}\right)$ at level $k=-4$ remains. This describes the spin sector. 
If $g_{\alpha}=-g_{m}>0$, the $\operatorname{osp}(2 \mid 2)$ current perturbation is irrelevant and we recover the previous description. Recall the original fermion $\Psi$, from which the density of states is computed, are related to $G$ and $\Psi^{\prime}$ by the chiral gauge transformation (4.25), so that the density of states is factorized as in eq.(4.27).

If $g_{\alpha}=-g_{m}<0$, the current-current perturbation is relevant. We may then propose that in the infrared the $\Psi^{\prime}-\beta^{\prime}$ system is described by the coset theory osp $(4 \mid 4)_{k=1} / \operatorname{osp}(2 \mid 2)_{k=-2}$, which is equivalent to an $s u(2)$ model at level zero. As a consequence, the fermions $\Psi^{\prime}$ shall flow in the infrared to fields with scaling dimension opposite to that of $G$, and the original fermions $\Psi$ shall flow to fields with zero scaling dimension. This suggests that in this case the density of states is finite and regular at zero energy.

\section{RG PHASE DIAGRAM}

In this section, we return to the general model with $g_{\alpha}+g_{m} \neq 0$, and describe the global features of the phase diagram based on the one-loop RG equations (3.10).

Our method consists in extracting the asymptotics of the RG trajectories by looking for directions in the coupling constant space which are preserved by the RG flow. Then, to analyze whether these asymptotic trajectories are attractive or not, stable or unstable, we project the RG flow onto the sphere and study the vector field thus obtained. This will allow us to point out the special role played by perturbations along a so-called strange direction.

Let us first look for lines in the coupling constant space, with coordinates $g=\left(g_{A}, g_{\alpha}, g_{m}\right)$, which are preserved by the RG flow and which pass through the origin. These correspond to trajectories which are straight lines and therefore for which the RG velocity field $\dot{g}=\beta(g)$ is co-linear to the vector $g$. The equations for these fixed line trajectories are thus:

$$
\beta(g) \wedge g=0
$$

The above equation is equivalent to finding solutions of the RG equations of the form $g^{i}(t)=x^{i} \lambda(t)$ where $x^{i}$ are constants independent of the RG time $t=\log l$ and $d \lambda(t) / d t=\lambda^{2}$. Substituting this into (3.6), one finds

$$
x^{i}=-C_{j k}^{i} x^{j} x^{k}
$$

where as before $C_{j k}^{i}$ are the operator product coefficients. With the linear relation $g^{i}=x^{i} \lambda$ among the couplings, the effective action contains a single running coupling constant:

$$
S_{\text {eff }}=S_{c f t}+\lambda \int \frac{d^{2} x}{2 \pi} \mathcal{O}_{x}, \quad \mathcal{O}_{x}=\sum_{i} x^{i} \mathcal{O}_{i}=x^{A} \mathcal{O}_{A}+x^{\alpha} \mathcal{O}_{\alpha}+x^{m} \mathcal{O}_{m}
$$

For $x^{i}$ a solution to (5.2), the operator $\mathcal{O}_{x}$ closes on itself under operator product expansion:

$$
\mathcal{O}_{x}(z, \bar{z}) \mathcal{O}_{x}(0) \sim-\frac{1}{z \bar{z}} \mathcal{O}_{x}(0)
$$

To lowest order the $\beta$ eta function for this specific perturbation is $\beta_{\lambda}=\lambda^{2}+\cdots$

There are six solutions to eqs.(5.1) (or eqs. (5.2)). They are: 


$$
\begin{aligned}
& A \equiv \quad\left(g_{A} \neq 0, g_{\alpha}=0, g_{m}=0\right) \\
& B \equiv \quad\left(g_{A}=0, g_{\alpha}=0, g_{m} \neq 0\right) \\
& C \equiv\left(g_{A}=0, g_{\alpha}+g_{m}=0\right) \\
& D \equiv\left(g_{\alpha}+g_{m}=0, g_{A}+2 g_{\alpha}=0\right) \\
& E \equiv\left(6 g_{\alpha}=-3(1+\sqrt{2}) g_{A}=-2(1+2 \sqrt{2}) g_{m}\right) \\
& F \equiv\left(6 g_{\alpha}=3(\sqrt{2}-1) g_{A}=2(2 \sqrt{2}-1) g_{m}\right)
\end{aligned}
$$

The two first lines correspond to the simple models with only random gauge potential [16],6] or only random mass [17 19]. The third and fourth ones correspond to the case we discuss at length in section IV. Contrary to the first four solutions, the last two do not seem to have an obvious simple algebraic interpretation 2 .

These six solutions correspond to twelve possible stable directions because we have to choose an orientation on the line. Only three of them are in the domain of positive coupling constants $g_{A}>0, g_{\alpha}>0$ and $g_{m}>0$. The first two, which are the solution $A$ with $g_{A}>0$ and the solution $B$ with $g_{m}>0$ are in the border of that domain. The third one, which is the solution $F$ with $g_{\alpha}>0$, sits in the middle of the domain of positive $g$ 's. We shall call this solution the strange direction.

To analyze deeper the RG flow let us now project it onto the sphere. This is worth doing since the beta functions are homogeneous. Since there are three coupling constants, we may parameterize them with two angles, that we shall denote $\theta_{1}$ and $\theta_{2}$, and the radial coordinates $\rho, \rho^{2}=g_{A}^{2}+g_{\alpha}^{2}+g_{m}^{2}$. The RG equations (3.10 may then be written as two equations for the angular variables

$$
\dot{\theta}_{j}=\rho \beta_{j}\left(\theta_{1}, \theta_{2}\right)
$$

together with one equation for the radial variable:

$$
\dot{\rho}=\rho^{2} \beta_{\rho}\left(\theta_{1}, \theta_{2}\right)
$$

The explicit expressions for the vector fields $\beta_{j}$ or $\beta_{\rho}$ are easy to find. In the angular equations (5.11) we may absorb the factor $\rho$ into a redefinition of the parametrization of the RG trajectories. This does not change the topology of the RG curves but only the speed at which the RG trajectories flow on these curves. So we shall analyze the vector field $\dot{\theta}_{j}=\beta_{j}\left(\theta_{1}, \theta_{2}\right)$ on the sphere. See figure 1 .

The vector field $\beta_{j}$ has twelve zeroes which correspond to the twelve fixed directions (5.5-5.10). We can compute the sign of $\dot{\rho}$ in these directions to know whether these straight RG trajectories are escaping to infinity $(\dot{\rho}>0)$ or are flowing back to the origin $(\dot{\rho}<0)$. To decipher whether the fixed directions are attractive or not we have to analyze whether the corresponding zero of the vector field $\beta_{j}$ on the sphere is attractive or not. For that we linearize the vector field $\beta_{j}$ on the sphere at its zeroes and compute its eigenvalues. Positive

\footnotetext{
${ }^{2}$ The operator $\mathcal{O}_{x}$ is bilinear in the currents, $\mathcal{O}_{x}=J^{A} d_{A B} \bar{J}^{B}$ with $J^{A}$ the $\operatorname{osp}(4 \mid 4)$ currents and $d_{A B}$ some bilinear form. The condition (5.4) is then: $f_{I}^{A B} d_{A N} d_{B M} f_{K}^{N M}=d_{I K}$. The algebraic interpretation of that equation is not clear to us.
} 
eigenvalues correspond to repulsive fixed directions, zero eigenvalues to locally marginal directions. For a fixed direction to be generically attractive, the two eigenvalues have to be non positive; otherwise to be attracted to the fixed direction requires fine tuning. The result is:

$$
\begin{aligned}
& A_{+}\left(g_{A}>0\right) \equiv \dot{\rho}>0 \text { and } \text { repulsive } \\
& A_{-}\left(g_{A}<0\right) \equiv \dot{\rho}<0 \text { and repulsive } \\
& B_{-}\left(g_{m}>0\right) \equiv \dot{\rho}<0 \text { and repulsive } \\
& B_{+}\left(g_{m}<0\right) \equiv \dot{\rho}>0 \text { and attractive } \\
& C_{-}\left(g_{\alpha}>0\right) \equiv \dot{\rho}<0 \text { and repulsive } \\
& C_{+}\left(g_{\alpha}<0\right) \equiv \dot{\rho}>0 \text { and attractive } \\
& D_{-}\left(g_{\alpha}>0\right) \equiv \dot{\rho}<0 \text { and attractive } \\
& D_{+}\left(g_{\alpha}<0\right) \equiv \dot{\rho}>0 \text { and } \text { repulsive } \\
& E_{-}\left(g_{\alpha}>0\right) \equiv \dot{\rho}<0 \text { and repulsive } \\
& E_{+}\left(g_{\alpha}<0\right) \equiv \dot{\rho}>0 \text { and repulsive } \\
& F_{+}\left(g_{\alpha}>0\right) \equiv \dot{\rho}>0 \text { and attractive } \\
& F_{-}\left(g_{\alpha}<0\right) \equiv \dot{\rho}<0 \text { and repulsive }
\end{aligned}
$$

The indices \pm refer to trajectories flowing to infinity or back to the origin.

There are only four fixed directions which are generically attractive: they stand on the directions $B_{+}, C_{+}, D_{-}$and on the strange direction $F_{+}$. They all are asymptotes to RG trajectories flowing to infinity except $D_{-}$which corresponds to asymptotic direction of trajectories looping back to the origin.

Let us pause to reconsider the case $g_{\alpha}+g_{m}=0$ is this language. In that case the solutions of the RG equations are $1 / g_{A}-1 / g_{A}^{0}=-2 t$ and $1 / g_{\alpha}-1 / g_{\alpha}^{0}=4 t$. If $g_{A}^{0}>0$ and $g_{\alpha}^{0}>0$, then $g_{A}$ increases whereas $g_{\alpha}$ decreases. These trajectories are asymptotic to the direction $A$ since $g_{A}$ blows up at a finite value of $t$ at which $g_{\alpha}$ is finite. However the blow up times are not physical since for $g_{A}$ large enough the one-loop analysis is no more valid and one has to relies on the non-perturbative analysis done in previous section. If $g_{A}^{0}>0$ but $g_{\alpha}^{0}<0$, then both couplings increase in magnitude. Which one blows up first depends on the initial data. The asymptotic trajectories are then either the direction $A$ or $C$ depending if $g_{A}^{0}+2 g_{\alpha}^{0}$ is positive or negative, so that the line $g_{A}+2 g_{\alpha}=0$ is a separatrix in the phase diagram. On contrary, if $g_{A}^{0}<0$ but $g_{\alpha}^{0}>0$, the couplings decrease and flow back towards the origin along the direction $D$.

The strange direction $F_{+}$is the asymptotic direction for all trajectories starting initially with positive coupling constants $g_{\alpha, m, A}$, except those which are fine tuned to be in the direction $A$ or $B$. The domain $g_{j}>0$ is stable under one-loop RG: no trajectories can escape from it. The fact that there is one and only one asymptotic direction in the domain of positive coupling constants reflects the universality of that behavior: whatever the values of the initial coupling constants, the system flows along this direction in the infrared. The strange direction is hence the one which should be relevant to the description of the low energy behavior of the $s u(2)$ random Dirac operators, e.g. the network model described in section II. Furthermore, since $\dot{\rho}>0$ for the strange direction, it is a strongly coupled system. 
In summary, based on the one-loop RG equations, it appears the generic network model is in a different universality class than the one fine-tuned to the line $g_{\alpha}+g_{m}=0$. However it certainly remains a possibility that the higher loop corrections can spoil the above analysis.

\section{DISCUSSION}

In summary, we have shown that the network model with the identification of couplings $g_{\alpha}+g_{m}=0$ exhibits a spin-charge separation in the effective disorder averaged theory, and this allows a precise identification of the infrared fixed point as the coset $\operatorname{ssp}(4 \mid 4)_{1} / s u(2)_{0}$. This coset conformal field theory has some novel features in that it possesses an $\operatorname{osp}(2 \mid 2)_{-2}$ supercurrent algebra symmetry, but due to the non-factorizability of the Hilbert space, it is not identical to the $\operatorname{ssp}(2 \mid 2)$ theory defined by the Sugawara construction. The resulting critical exponents agree with the predictions based on percolation [ $⿴$ ] and the numerical simulations of the super spin chain studied in [3]. Based on the one-loop renormalization group, we have argued that the network model without the constraint $g_{\alpha}+g_{m}=0$ is in a different universality class.

How our analysis relates to percolation remains an interesting open question. There appear to be two possibilities. One is that the 1-loop strange direction we described in section $\mathrm{V}$ survives to higher loops and indeed corresponds to percolation; our coset theory $\operatorname{osp}(4 \mid 4)_{1} / s u(2)_{0}$ is then the wrong fixed point. Another possibility is that the higher loop corrections actually restore the symmetry to the line $g_{\alpha}+g_{m}=0$, and the coset $\operatorname{osp}(4 \mid 4)_{1} / \operatorname{su}(2)_{0}$ is a new description of percolation with $\operatorname{osp}(2 \mid 2)$ symmetry. The latter possibility can be investigated by comparing the conformal blocks of percolation with those computed in [20].

It would be interesting to construct explicitly the continuum field theory corresponding to the super spin chain along the lines of [21]. It is important to understand the role of the global $\operatorname{osp}(2 \mid 2)$ symmetry of the spin chain if this continuum limit indeed corresponds to percolation.

\section{ACKNOWLEDGMENTS}

We thank A. Ludwig, H. Saleur and D. Serban for discussions, and A. La Belière for spirited encouragement. D.B. was supported in part by the CNRS, by the CEA and the European TMR contract ERBFMRXCT960012.

\section{APPENDIX: SPIN-CHARGE SEPARATION OF THE STRESS TENSOR.}

We give here some details on the proof of eq.(4.12) for the energy momentum tensor. Recall that the Sugawara construction of the energy momentum tensor in terms of the currents $J^{a}$ is:

$$
T(z)=\kappa \lim _{w \rightarrow z}\left(J^{a}(w) C_{a b} J^{b}(z)-k \frac{C_{a b} \delta^{a b}}{(z-w)^{2}}\right)
$$


with $C_{a b}$ the quadratic Casimir and the normalization constant $\kappa$ is chosen such that the currents have conformal dimension one. In practice $T$ is computed by extracting the regular term in product $J^{a}(z) C_{a b} J^{b}(0)$.

For the $\operatorname{osp}(2 \mid 2)$ algebra at level -2 this gives:

$$
T_{o s p(2 \mid 2)_{-2}}=\frac{1}{8}\left(J^{2}-H^{2}-\frac{1}{2}\left(J_{-} J_{+}+J_{+} J_{-}\right)+\left(S_{+} S_{-}-S_{-} S_{+}\right)+\left(\widehat{S}_{-} \widehat{S}_{+}-\widehat{S}_{+} \widehat{S}_{-}\right)\right)
$$

The $\operatorname{osp}(2 \mid 2)$ currents are given in eq.(4.1) in terms of the $\beta-\psi$ system. This Sugawara energy momentum tensor can thus be expressed in terms of these bosonic and fermionic fields. A simple computation yields:

$$
\begin{aligned}
& T_{o s p(2 \mid 2)_{-2}}=\frac{1}{8}\left(\partial_{z} \psi_{-} \psi_{+}+\partial_{z} \psi_{+} \psi_{-}+\partial_{z} \beta_{-} \beta_{+}-\partial_{z} \beta_{+} \beta_{-}-4\left(\psi_{-} \beta_{+}\right)\left(\beta_{-} \psi_{+}\right)\right. \\
& \left.+3\left(\psi_{-} \psi_{+}\right)^{2}-\left(\beta_{-} \beta_{+}\right)^{2}+2\left(\psi_{-} \psi_{+}\right)\left(\beta_{-} \beta_{+}\right)\right)
\end{aligned}
$$

Similarly, with the normalization (3.7) for the Pauli matrices, the $s u(2)$ Sugawara tensor is:

$$
T_{s u(2)_{0}}=\frac{1}{8} L^{a} L^{a}
$$

with $L^{a}$ defined in eq.(4.10). Again with the help of the identity (4.5), this may be written in terms of the $\beta-\psi$ system as:

$$
\begin{gathered}
T_{s u(2)_{0}=\frac{1}{8}(}\left(3\left(\partial_{z} \psi_{-} \psi_{+}+\partial_{z} \psi_{+} \psi_{-}+\partial_{z} \beta_{-} \beta_{+}-\partial_{z} \beta_{+} \beta_{-}\right)+4\left(\psi_{-} \beta_{+}\right)\left(\beta_{-} \psi_{+}\right)\right. \\
\left.-3\left(\psi_{-} \psi_{+}\right)^{2}+\left(\beta_{-} \beta_{+}\right)^{2}-2\left(\psi_{-} \psi_{+}\right)\left(\beta_{-} \beta_{+}\right)\right)
\end{gathered}
$$

Adding both pieces we get:

$$
T_{o s p(2 \mid 2)_{-2}}+T_{s u(2)_{0}}=\frac{1}{2}\left(\partial_{z} \psi_{-} \psi_{+}+\partial_{z} \psi_{+} \psi_{-}+\partial_{z} \beta_{-} \beta_{+}-\partial_{z} \beta_{+} \beta_{-}\right)
$$

This is the energy momentum tensor $T$ for the conformal field theory $S_{c f t}$. 


\section{REFERENCES}

[1] V. Kagalovsky, B. Horovitz, Y. Avishai and J. T. Chalker, Phys. Rev. Lett. 82 (1999) 3516.

[2] T. Senthil and M. P. A. Fisher, cond-mat/9810238.

[3] T. Senthil, J. B. Marston and M. P. A. Fisher, Phys. Rev. B 60 (1999) 4245.

[4] I. A. Gruzberg, A. W. W. Ludwig, and N. Read, Phys.Rev.Lett. 82 (1999) 4524.

[5] A. Altland and M. Zirnbauer, Phys. Rev. B55 (1997) 1142; M. Zirnbauer, J. Math. Phys. 37 (1996) 4986.

[6] D. Bernard, (Perturbed) conformal field theory applied to 2d disordered systems: an introduction, hep-th/9509137, in proceeding of Cargèse conference, July 1995, Nato ASI, series B: physics vol.362, Plenum Press.

[7] C. Mudry, C. Chamon, and X.-G. Wen, Nucl.Phys. B466 (1996) 383-443, condmat/9509054.

[8] D. Friedan, E. Martinec and S. Shenker, Nucl. Phys. B271 (1986) 93.

[9] A. B. Zamolodchikov, Int. J. Mod. Phys. A4 (1989) 4235.

[10] S. Guruswamy, A. LeClair and A. W. W. Ludwig, cond-mat/9909143.

[11] V. Knizhnik and A. Zamolodchikov, Nucl. Phys. B247 (1984) 83.

[12] P. Bowcock, M. Hayes and A. Taormina, hep-th/9705234.

[13] Z. Maassarani and D. Serban, Nucl.Phys. B489 (1997) 603-625.

[14] J.-S. Caux, I. Kogan, A. Lewis, and A. M. Tsvelik, Nucl.Phys. B489 (1997) 469-484, hep-th/9606138.

[15] K. Gawedzki and A. Kupiainen, Nucl. Phys. B320 (1989) 625.

[16] A. Nersesyan, A. Tsvelik and F. Wegner, Phys. Rev. Lett. 72 (1994) 2628.

[17] V. Dotsenko and Vl. Dotsenko, Adv. Phys. 32 (1983) 129.

[18] A. Ludwig, Nucl. Phys. B 330 (1990) 639.

[19] R. Shankar, Phys. Rev. Lett. 58 (1987) 2466.

[20] J.-S. Caux, N. Taniguchi, and A. M. Tsvelik, Nucl.Phys. B525 (1998) 671-696, condmat/9801055.

[21] H. Saleur, solv-int/9905007, Nucl.Phys. B578 (2000) 552-576. 


\section{FIGURES}

FIG. 1. The vector field $\beta_{j}$ on the sphere. We choose the coordinates $4 g_{\alpha}=\cos q$, $2\left(g_{\alpha}+g_{m}\right)=\sin q \cos p$ and $2 g_{A}=\sin q \sin p$ with $q \in[0, \pi]$ and $p \in[0,2 \pi]$.

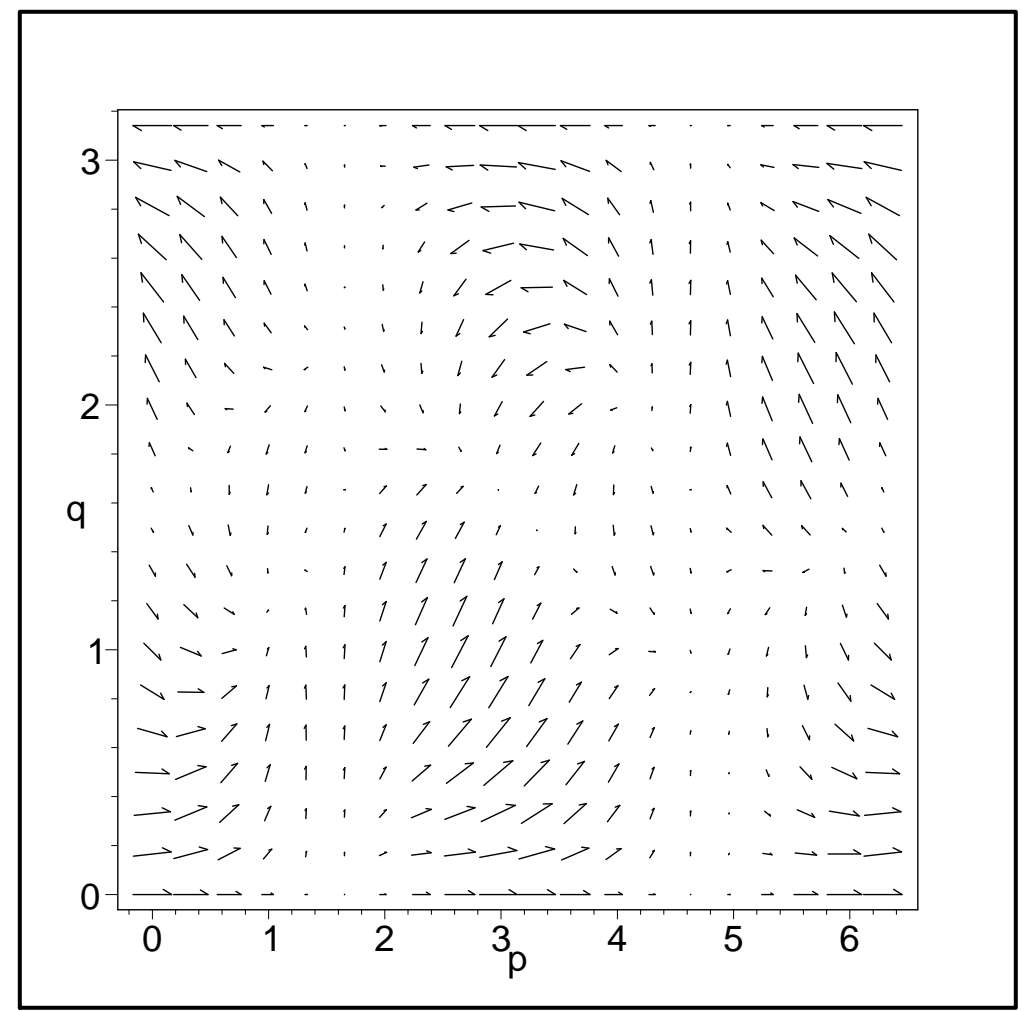

FIG. 2. Structure of the indecomposable $\operatorname{osp}(2 \mid 2)$ representation formed by the field appearing in $\widetilde{\mathcal{O}}, L_{S}=L_{f}+L_{b}=L$ and $L_{A}=L_{f}-L_{b}$. The solid arrows describe the action of $S_{-}$and the dashed ones the action of $\widehat{S}_{-}$.



